\title{
Laser treatment of twin-to-twin transfusion syndrome (TTTS): preliminary findings from a tertiary center in Greece
}

\section{Georgios Papaioannou, Nikolaos Evangelinakis, Nikolaos Papantoniou}

$3^{\text {rd }}$ Department of Obstetrics and Gynecology, Attikon Hospital, National and Kapodistrian University of Athens, Greece

Corresponding Author

Nikolaos Papantoniou, MD, PhD, V. Sofias Ave 82, Athens 11528, e-mail: npapant@gmail.com

\section{Abstract}

Background: Twin- to-twin transfusion syndrome (TTTS) affects approximately $15 \%$ of monochorionic twin pregnancies with an estimated incidence of 1 in 1,600 pregnancies. The gold standard of care is considered to be Fetoscopic Laser Photocoagulation Therapy (FLPT).

Objective: To present our experience with FLPT in a preliminary prospective observational study.

Materials and methods: Fourteen patients who underwent FLPT in the Fetal Maternal Medicine Unit of Attikon University Hospital, between January 2017 and August 2018. Fetoscopic laser photocoagulation was offered to all patients with pregnancies complicated by TTTS (Quintero stage $>2$ ), before the completion of 26 weeks of gestation.

Results: The mean gestational age at diagnosis was $22.2 \pm 2.6$ weeks, at surgery $23.1 \pm 1.9$ weeks and at birth $29.8 \pm 3.3$ weeks. Mean interval from surgery to delivery was $6.6 \pm 3.1$ weeks and mean neonatal birthweight was $1426 \pm 408$ grams. Mean recipient's weight at delivery was $1652 \pm 283 \mathrm{gr}$, whereas mean donor's weight at delivery was $1299 \pm 402$ gr. Concerning fetal viability, ten $(71,4 \%)$ donor fetuses survived to full term whereas $4(28,6 \%)$ donor fetuses died in utero. The survival rate of recipient fetuses reached $85,7 \%$ which is very good according to the literature. In total 22 fetuses $(78,6 \%)$ were alive at birth and survived the first 6 months of life and in 6 cases an intrauterine demise occurred $(21,4 \%)$. In 10 pregnancies $(71,4 \%)$ both fetuses survived, in $2(14,3 \%)$ both died and in 12 pregnancies $(85,7 \%)$ there was at least one survivor.

Conclusion: These preliminary results support the use of FPLT in Greece as the percentage of live neonates that was reached is comparable to those published in larger studies in the international literature. We are confident that with increasing experience and new instrumentation, these results will become even better and this service will accept more cases all over Greece.

Key words: TTTS; FPLT; laser; twin-to-twin transfusion; Greece 


\section{Introduction}

Twin- to-twin transfusion syndrome (TTTS) affects approximately $15 \%$ of monochorionic twin pregnancies with an estimated incidence of 1 in 1,600 pregnancies and is considered as the most serious complication of multiple gestation ${ }^{1}$. The syndrome is characterized by an unequal blood flow distribution between the fetuses in the placenta via unidirectional deep arteriovenous vascular anastomoses. As a result blood is transferred from one twin (donor) to the other (recipient) and the donor twin presents with growth retardation, oligohydramnios and abnormal findings on umbilical artery (UA) Doppler ultrasonography ${ }^{2}$. In contrast, the recipient twin presents with polyhydramnios, signs of congestive heart failure and abnormal findings on venous Doppler ultrasonography (reverse flow in the ductus venosus [DV] or pulsatile flow within the umbilical vein) and if untreated progresses into hydrops and intrauterine demice ${ }^{3}$.

The prognosis of pregnancies complicated by TTTS is extremely poor as it is estimated that the perinatal mortality rate exceeds $90 \%$ with the donor twin being at higher risk of death compared to the recipient ${ }^{4,5}$. Also, one third of the surviving fetuses have neurodevelopmental morbidity as a result of prematurity, placental hypo perfusion and other perinatal adverse events ${ }^{5,6}$.

Several treatment modalities have been proposed for the management of TTTS including serial amnioreduction, septostomy of the intramniotic membrane and umbilical cord ligation. However, their efficacy in optimizing the antenatal fetal mortality and morbidity rates is to the least controversial. The first prospective randomized controlled trial, that compared serial amnio-drainage to laser coagulation of chorionic plate anastomoses (Fetoscopic Laser Photocoagulation Therapy, FLPT) was published in 2004 by Senat et $\mathrm{al}^{7}$. The authors concluded that endoscopic laser therapy significantly increased the survival rates of at least one twin to 28 days of age and the study set a boundary for a new era of fetoscopic treatment of the syndrome. Since then, several studies were published in this field, however, to date the experience in the Greek setting remained scarce and anecdotal. The purpose of the present study is to present our experience which is based in a preliminary case series of patients treated in a tertiary university hospital in Greece.

\section{Materials and Methods}

Our study is based in a prospective case series of 14 patients who underwent FLPT in the Fetal Maternal Medicine Unit of Attikon University Hospital, between January 2017 and August 2018. FLPT was offered to all patients with pregnancies complicated by TTTS (Quintero stage $>2$ ), before the completion of 26 weeks of gestation. All procedures were carried out by experienced maternal-fetal medicine specialists that received special training on FLPT.

Gestational age was determined by the crownrump length (CRL) measured at $11-13^{+6}$ weeks of gestation. In case this was not feasible dating was based on the head circumference of the recipient twin. All scans were performed in a Voluson E6 ultrasound system (GE Medical System, Milwaukee, WI, USA).

After the diagnosis of TTTS, treatment options were offered to the patients. Patients with Quintero stage II or more were offered FLPT. All cases were referrals and the majority were Stage III. Cases that were previously treated with amnioreduction and exceeded the gestational age of 26 weeks, were subjected in repeat amnioreduction or expectant management. Patients were admitted on the day of the procedure and post operatively they were given the option of discharge 1- 2 days later or remain hospitalized for the following week for close fetal monitoring, especially those whose permanent residence was remote from Athens or specialized 
MFM Centers.

The procedure was performed in the day-case surgery operating theatre, under local anesthesia. Antibiotics were administered prior and after the procedure (iv cefazolin $1 \mathrm{~g}$ immediately prior to skin incision and every 8 hours for two days). An ultrasound examination was performed prior to the procedure to determine insertion of the cords of both fetuses, the distance between them and the location of the placenta. Instruments used included a 2-mm fetoscope (HOPKINS® II Straight Forward Telescope $0^{\circ}$ 26008AA; Karl Storz, Tuttlingen, Germany), a diode laser with a 600- $\mu \mathrm{m}$ laser fibre (Dornier Medilas D MultiBeam; Dornier MedTech, Wessling, Germany), vascular access trocar and cannula (Terumo Radifocus 11F; Terumo Corporation, Tokyo, Japan). The $2 \mathrm{~mm}$ trocar was inserted in the amniotic cavity of the recipient twin under sonographic guidance and was directed to the middle of an imaginary line connecting the 2 umbilical cord insertions.

Anastomoses were identified and then coagulated with continuing beam of Nd-YAG laser and the energy was set at 25-35 $\mathrm{W}$ depending on the diameter of each targeted vessel. In order to maintain adequate vision during the procedure and to cool the laser beam Hartmann's solution was infused through a side port of the fetoscope. After laser surgery was completed, an amnioreduction followed in order to reduce amniotic fluid of the recipient twin, until the maximum vertical pocket was $<8 \mathrm{~cm}$. Fetal viability was assessed during and after the procedure.

Abdominal incision was reapproximated either with sterile strips or with a Nylon 1-0 suture. The patient was then admitted for an overnight stay and started on vaginal suppositories of indomethacin or diclophenac sodium. At postoperative day $1 \mathrm{a}$ transvaginal ultrasound was performed and an Arabin pessary was placed if the cervical length was measured $<12 \mathrm{~mm}$.

Afterwards, they were followed up closely (at least twice a week) either at our unit or at experienced Fetal Medicine units at their place of permanent residence. In all follow up scans Doppler studies of the umbilical arteries (UA), ductus venosous (DV) and middle cerebral artery (MCA) were performed and deepest vertical pool and discordance of the fetal weights were measured. All patients were followedup for post-operative complications and to assess pregnancy outcomes. Neonatal outcome was also followed up until the first year of life to evaluate for potential neurodevelopmental anomalies.

\section{Results}

Fourteen (14) pregnancies complicated by TTTS were referred for evaluation and were treated between September 2016 and August 2018 (Table 1 ). The mean gestational age at referral was $22.2 \pm$ 2.6 weeks, at surgery $23.1 \pm 1.9$ weeks and at birth $29.8 \pm 3.3$ weeks. Mean interval from surgery to delivery was $6.6 \pm 3.1$ weeks and mean neonatal birthweight was $1426 \pm 408$ grams. Mean recipient's weight at delivery was $1652 \pm 283 \mathrm{gr}$, whereas mean donor's weight at delivery was $1299 \pm 402 \mathrm{gr}$ (Table 2).

The majority of cases treated with laser photocoagulation were Quintero stage III ( $\mathrm{n}=10,71,4 \%)$, three cases were of Quintero stage $2(n=3,21,4 \%)$ and one case of Quintero stage 1. In most cases the placenta was posterior or predominantly posterior $(\mathrm{n}=8,57,1 \%)$.

Separating membrane remained intact in all cases and there were no cases with intrauterine infection. In one case bleeding occurred during FLPT due to laceration of a vessel but further laser coagulation of the upstream of this vessel, succeeded in creating a clot ceasing the bleeding.

Table 1. Survival outcomes until 1 year of age.

$\begin{array}{lcccc}\text { Survival } & \mathbf{2} & \mathbf{1} & \mathbf{0} & \text { At Least 1 } \\ \text { Outcome } & 9 & 3 & 2 & 12 \\ \text { \% survival } & 64,3 & 21,4 & 14,3 & 85\end{array}$


Table 2. Neonatal characteristics

$\begin{array}{lccc} & \text { All alive } & \text { Recipients } & \text { Donors } \\ \text { Birthweight } & 1425.45 \pm 407.9 & 1651.82 \pm 282.59 & 1299.38 \pm 401.67 \\ \text { Days in NICU } & 34.88 \pm 17.38 & 24.43 \pm 13.13 & 36.5 \pm 14.18\end{array}$

There were no cases with preterm premature rupture of membranes (PPROM) 24 hours following after the procedure and in the majority of cases $(85,7 \%)$ membranes remained intact until delivery. In 2 cases PPROM occurred 3 and 4 weeks postoperatively at $28^{\text {th }}$ and $30^{\text {th }}$ week of gestation. No cases of maternal bleeding or infection were detected.

Concerning fetal viability, ten $(71,4 \%)$ donor fetuses survived to full term whereas $4(28,6 \%)$ donor fetuses died in utero. The survival rate of recipient fetuses reached $85,7 \%$ which is very good according to the literature. In total 22 fetuses $(78,6 \%)$ were alive at birth and survived the first 6 months of life and in 6 cases an intrauterine demise occurred $(21,4 \%)$. In 10 pregnancies $(71,4 \%)$ both fetuses survived, in $2(14,3 \%)$ both died and in 12 pregnancies $(85,7 \%)$ there was at least one survivor.

\section{Discussion}

Twin to twin transfusion syndrome, mainly occurs in monochorionic twin pregnancies [MCTP]. The syndrome has been described in the Bible and in medieval ages with the birth of two dead fetuses or stillbirths, characterized by excessive difference in size and hemoglobin. Severe hydramnios and hydropic appearance for the Recipient $[\mathrm{R}]$ and intrauterine growth restriction and anydramnio for the Donor[D], were the common phenotypic and clinical findings reported. The etiology of the syndrome is well known today and is due to hemodynamic imbalance between the two fetal circulations, caused mainly by arteriovenous anastomoses. Deep arteriovenous anastomoses are responsible for the syndrome leading the blood flow from the Donor to the Recipient. The superficial arterio-arterial anas- tomoses and vein to vein anastomoses are bidirectional and play a compensative role. Under certain circumstances they may perform as unidirectional and worsen the hemodynamics ${ }^{8}$.

TTTS presents a major challenge for treatment in Perinatal Medicine, since two otherwise healthy fetuses if untreated will be lost, in more than $80 \%$ of cases. Thus, antenatal treatment is essential for ameliorating the perinatal outcome. Previous approaches to the syndrome were umbilical cord ligation, septostomy and amnioreduction, however, their efficacy in optimizing pregnancy outcomes does not seem to be comparable to FLPT ${ }^{9,10}$. Specifically, umbilical cord ligation and septostomy were soon abandoned following their institution, due to complications such as a priory survival rate of $50 \%$ or less, in cases treated with umbilical cord ligation and demonstration of amniotic band syndrome in cases treated with septostomy. Since 2004, and the publication of the EUROFETUS RCT (7), the majority of maternal fetal medicine institutes in Europe and Northern America adopted laser as the optimal treatment, restricting serial amnioreduction in cases detected after the completion of the $26^{\text {th }}$ week of gestation.

In Greece FLPT remained at large unavailable and scarce anecdotal cases have been reported, without validation of survival outcomes. Considering Greece`s birth rates, approximately 70.000 deliveries, the incidence of TTTS is estimated that 80 cases present annually with the syndrome, thus pointing the need for specialized medical care in this field, as all these cases were directed to seek treatment abroad. Given Greece's economic recession, it is easily understandable that several cases did not have the 
required background to cover the expenses and in the past were treated with serial amnioreductions. An interesting finding of our study was a relatively small percentage of couples that decided to terminate the pregnancy $(2 / 21)$. The main reason they decided to terminate was the fear of neonatal complications, such as neurodevelopmental morbidity. In our institution the option of termination was not offered. The later underlines the necessity of the institution of FLPT in the Greek NHS with good and trustworthy results.

The majority of our cases were Quintero III(10/14 $-71,4 \%)$, which shows that they were either late diagnosis or severe cases of acute TTTS. Several surgical alternatives of FLPT approaches have been introduced during the last years including selective, sequential and Solomon technique ${ }^{11-13}$. In selective technique only the anastomotic vessels are coagulated whereas in Solomon technique coagulation is applied between the anastomoses, resulting in a complete separation of the placenta. In sequential technique -a variation of selective technique- anastomoses from donor to recipient are coagulated first and the anastomoses from recipient to donor are coagulated afterwards, thus allowing blood to be transfused to the donor ${ }^{8,11,12}$. Recently the Dutch group who published the Solomon study, evaluated the impact of laser on placental surface and showed that greater damage caused by Solomon technique may be responsible for increased preterm births, which needs further clinical evaluation ${ }^{14}$.

In our series the FLPT was performed using the selective technique and only in the last 3 cases we used the Solomon technique, since it needs higher expertise and reaching an appropriate level in the learning curve is needed to perform it. This finding is in accordance to the observation of a worldwide survey which reported that high volume centers $>20$ cases treated per year) prefer the Solomon technique, whereas low-volume centers tend to stick with the selective ablation of anastomotic vessels ${ }^{15}$. Our results are almost similar with recent literature evaluation data of FLPT during the past 25 years, where 1 twin survival raised from 35 to $65 \%$ and double twin survival from 70 to $88 \%{ }^{13}$. We presented an $85 \%$ one twin survival and $65 \%$ double twin survival and believe that this is due to the fact that all procedures were carried out by experienced physicians in MFM, under sterile conditions, following meticulously the guidelines as presented by Peeters et $\mathrm{al}^{16}$.

The incidence of PPROM in our case series was far less compared to that of previous studies in the field that reported a similar survival ${ }^{17}$; however, the relatively small number of cases presented restrict safe conclusions concerning this outcome. Nevertheless, the use of a smaller diameter trocar ( $2 \mathrm{~mm})$ might have contributed to this and this remains an unanswered parameter to be investigated in the future. Another explanation is that in all cases with short cervix $(<15 \mathrm{~mm})$ we applied Arabin vaginal pessary. There is an observational study suggesting that emergency cerclage prolongs pregnancy and improves perinatal outcome ${ }^{18}$. On the other hand there are studies showing lower rate of preterm birth after placement of an Arabin cervical pessary ${ }^{19,20,21}$. Arabin pessary has the advantages over emergency cerclage that does not require anesthesia, is easy to be applied in outpatient setting, can easily be removed at any time and does not bear increased risk of infection or rupture of membranes. The PECEP laser randomized controlled trial is currently under way aiming to answer whether cervical pessary prolongs pregnancy in cases of TTTS after fetoscopy regardless of cervical length ${ }^{22}$.

Antibiotics were administered in all cases 1 hour prior to laser procedure and for the following 4 days. This is another parameter that may have positively influenced our good results concerning PROM.

We had 2 cases with IUDs [ 4 twins]. The first case was with posterior placenta, large vessels and easy 
strait forward procedure, to us unexplained. The other set of twins was complicated by placental abruption 4 days after the procedure. Our total double fetal loss rate was $14.3 \%$ which is in accordance with current literature.

All cases were evaluated with Doppler measurements of the umbilical artery (UA), the middle cerebral artery(MCA) and the ductus venosus(DV) preoperative and at 48 hours following the procedure. Thereafter the follow-up was twice weekly as we believe that it is of crucial importance to monitor these fetuses closely until birth due to possible postoperative complications. In our series recurrent TTTS was observed in 1 case [7\%] following the selective technique and in 1 case TAPS [7\%] presented as a complication following the Solomon approach. Compared with reported incidence in the literature for the Solomon technique, we were on the medium range for TAPS [3vs16]. We followed up closely these cases with dopplers and delivered on time with no additional invasive procedures and no perinatal losses.

In general our perinatal losses were 2 pregnancies with IUDs [4 fetuses] and another two cases with single fetal demise which is compatible with recently published data ${ }^{13}$. It is noteworthy that we had no cases with cerebral palsy post laser and no cases with neurological morbidity during the first year of life.

\section{Conclusion}

We presented the first series of in utero treatment with FLPT in Greece and showed that our results were compatible with the literature, having a double twin survival of $71 \%$ and one twin survival $86 \%$. We are confident that with increasing experience and new instrumentation, these results will be better and this service will accept more cases all over our country.

\section{Conflicts of interest}

None to declare

\section{Sources of Funding}

None

\section{References}

1. Jain V, Fisk NM. The twin-twin transfusion syndrome. Clin Obstet Gynecol. 2004;47:181-202.

2. Galea P, Jain V, Fisk NM. Insights into the pathophysiology of twin-twin transfusion syndrome. Prenat Diagn. 2005;25:777-85.

3. Patel S, Quintero RA, Kontopoulos EV, Korst LM, Llanes A, Chmait RH. Abnormal Umbilical Artery Doppler Findings in the Recipient Twin Before Laser Surgery for Twin-Twin Transfusion Syndrome. Journal of Ultrasound in Medicine. 2015;34:843-6.

4. Salomon LJ, Ville Y. Twin-to-twin transfusion syndrome: diagnosis and treatment. Bull Acad Natl Med. 2008;192:1575-86; discussion 86-7.

5. Wagner S, Repke JT, Ural SH. Overview and Longterm Outcomes of Patients Born With Twin-toTwin Transfusion Syndrome. Reviews in obstetrics \& gynecology. 2013;6:149-54.

6. Li X, Morokuma S, Fukushima K, et al. Prognosis and long-term neurodevelopmental outcome in conservatively treated twin-to-twin transfusion syndrome. BMC pregnancy and childbirth. 2011;11:32-.

7. Senat MV, Deprest J, Boulvain M, Paupe A, Winer $\mathrm{N}$, Ville $\mathrm{Y}$. Endoscopic laser surgery versus serial amnioreduction for severe twin-to-twin transfusion syndrome. N Engl J Med. 2004;351:136-44.

8. Quintero RA, Kontopoulos E, Chmait RH. Laser Treatment of Twin-to-Twin Transfusion Syndrome. Twin Res Hum Genet. 2016;19:197-206.

9. Rossi AC, D’Addario V. Umbilical cord occlusion for selective feticide in complicated monochorionic twins: a systematic review of literature. Am J Obstet Gynecol. 2009;200:123-9.

10. Roberts D, Neilson JP, Kilby MD, Gates S. Interventions for the treatment of twin-twin trans- 
fusion syndrome. Cochrane Database Syst Rev. 2014:Cd002073.

11. Slaghekke F, Lopriore E, Lewi L, et al. Fetoscopic laser coagulation of the vascular equator versus selective coagulation for twin-to-twin transfusion syndrome: an open-label randomised controlled trial. Lancet. 2014;383:2144-51.

12. Akkermans J, Peeters SH, Klumper FJ, Middeldorp JM, Lopriore E, Oepkes D. Is the Sequential Laser Technique for Twin-to-Twin Transfusion Syndrome Truly Superior to the Standard Selective Technique? A Meta-Analysis. Fetal Diagn Ther. 2015;37:251-8.

13. Akkermans J, Peeters SH, Klumper FJ, Lopriore E, Middeldorp JM, Oepkes D. Twenty-Five Years of Fetoscopic Laser Coagulation in Twin-Twin Transfusion Syndrome: A Systematic Review. Fetal Diagn Ther. 2015;38:241-53.

14. Akkermans J, de Vries SM, Zhao D, et al. What is the impact of placental tissue damage after laser surgery for twin-twin transfusion syndrome? A secondary analysis of the Solomon trial. Placenta. 2017;52:71-6.

15. Akkermans J, Peeters SH, Middeldorp JM, et al. A worldwide survey of laser surgery for twin-twin transfusion syndrome. Ultrasound Obstet Gynecol. 2015;45:168-74.

16. Peeters SH, Akkermans J, Westra M, et al. Identification of essential steps in laser procedure for twin-twin transfusion syndrome using the Delphi methodology: SILICONE study. Ultrasound Obstet Gynecol. 2015;45:439-46.

17. Stirnemann J, Djaafri F, Kim A, et al. Preterm premature rupture of membranes is a collateral effect of improvement in perinatal outcomes following fetoscopic coagulation of chorionic vessels for twin-twin transfusion syndrome: a retro- spective observational study of 1092 cases. Bjog. 2018;125:1154-62.

18. Salomon LJ, Nasr B, Nizard J, Bernard JP, Essaoui M, Bussieres L, Ville Y. Emergency cerclage in cases of twin-to-twin transfusion syndrome with a short cervix at the time of surgery and relationship to perinatal outcome. Prenat Diagn. 2008;28:1256-1261.

19. Goya M, de la Calle M, Pratcorona L, Merced C, Rodó C, Muñoz B, et al. Cervical pessary to prevent preterm birth in women with twin gestation and sonographic short cervix: a multicenter randomized controlled trial (PECEP- twins) AJOG. 2016;214(2):145-152.

20. Arabin B, Halbesma JR, Vork F, Hubener M, van Eyck J. Is treatment with vaginal pessaries an option in patients with a sonographically detected short cervix? J Perinat Med. 2003;31:122-133.

21. Carreras E, Arévalo S, Bello-Muñoz JC, Goya M, Rodó C, Sánchez-Duran MA, Cabero L. Arabin cervical pessary to prevent preterm birth in severe twin-to-twin transfusion syndrome. Prenat Diagn. 2012;32:1-5.

22. Rodo C, Arevalo S, Lewi L, Couck I, Hollwitz B, Hecher K, Carreras E, Arabin cervical pessary for prevention of preterm birth in cases of twin-totwin transfusion syndrome treated by fetoscopic LASER coagulation: the PECEP LASER randomised controlled trial. BMC Pregnancy Childbirth. 2017 Aug 1;17(1):256). 